\title{
Calibração de Termohigrometro para Utilização em Pesquisas Microclimáticas
}

\section{Calibration Thermohigrometer for use in Research Microclimatic}

\author{
${ }^{1}$ Debora Aparecida Souza Guedes, ${ }^{2}$ Vera Cristina de Area Leão Borges \\ ${ }^{1}$ Engenheira Sanitarista e Ambiental - UFMT, Mestranda no Programa de Pós Graduação em Engenharia de \\ Edificações e Ambiental - PPGEA, Faculdade de Engenharia, Arquitetura e Tecnologia - FAET, Universidade \\ Federal de Mato Grosso - UFMT \\ ${ }^{2}$ Mestranda no Programa de PPGEA/ FAET - UFMT
}

RESUMO: Com o avanço das pesquisas ambientais e os estudos dos comportamentos climáticos, cada vez mais são requeridos dados climatológicos com alto grau de precisão, porém os custos com hardware e software nem sempre são satisfatórios. Os principais dados para pesquisas ambientais são as medidas de temperatura e umidade relativa, necessitando de sensores de alta acurácia. O Termo-higrômetro é um instrumento de medição das temperaturas interna e externa e da umidade relativa. Nesse contexto, o objetivo deste trabalho foi realizar a calibração de sensor termo-higrômetro para medir as variáveis em ambientes externos. A calibração do sensor foi realizada com equipamento similar certificado, denominado de Referência. Como resultado obteve-se um coeficiente de Correlação para temperatura $\mathrm{R}^{2}=0,9997$ entre os dados do sensor calibrado e o de Referência, e Coeficiente de Correlação para umidade $\mathrm{R}^{2}=0,9995$. A concordância obtida foi igual a 1 , concordância perfeita. Palavras-chave: Temperatura, Umidade Relativa, Comportamentos climáticos

\begin{abstract}
With the advancement of environmental research and studies of climate behavior, are increasingly required climatological data with high degree of accuracy, but the cost of hardware and software are not always satisfactory. The key data for environmental research are the measurements of temperature and relative humidity, requiring high accuracy sensors. The thermo- hygrometer is an instrument for measuring indoor and outdoor temperatures and humidity. In this context, the objective of this study was the thermo-hygrometer sensor calibration to measure the variables outdoors. The sensor calibration was performed with certified equipment similar called Reference. As a result, we obtained a correlation coefficient for $\mathrm{R}^{2}=0.9997$ temperature between the data of the calibrated sensor and reference, and correlation coefficient for moisture $\mathrm{R}^{2}=0.9995$. The resulting agreement was equal to 1 , perfect agreement.
\end{abstract}

Keywords: Temperature; Relative Humidity; Climatic behavior

\section{INTRODUÇÃO}

Com as mudanças climáticas houve a necessidade do avanço em pesquisas ambientais que avaliassem os comportamentos climáticos. A maior necessidade era dispor de medidas precisas dos parâmetros do microclima, como temperatura e umidade relativa (UR).

De acordo com Callejas (2014), os instrumentos de medição se difundiram largamente nas áreas de Ciências Naturais e nas áreas de Engenharia com objetivo de medir, controlar, atuar, monitorar e registrar variáveis físicas ambientais.

Estudos relacionados aos comportamentos do microclima têm sido amplamente estudados por possuir importância nos processos ambientais.

Segundo Jensen (2009), a temperatura é definida representação da energia das partículas da matéria em movimento aleatório.

Os sensores de temperatura possuem funções de transferências não lineares exigindo circuitos relativamente complexos, o que requer resistores de alta precisão e calibração (SRBINOVSKA et al., 2015 apud NEVES et al, 2015).

Umidade Reativa é a relação entre a quantidade de água existente no ar e o seu ponto de saturação (SILVA, 2009). 
Um dos instrumentos utilizados para a medição da umidade relativa é o higrômetro. Existem vários métodos para se medir UR, atualmente as medidas de são realizadas através das alterações produzidas pelo vapor d'água nas características elétricas de componentes inseridos em circuitos eletrônicos apropriados.

Nesse contexto, o objetivo desse trabalho foi calibrar termo-higrômetro para medir temperatura e umidade relativa em ambientes externos.

\section{MATERIAL E MÉTODOS}

Este experimento foi realizado na área urbana de Cuiabá, no prédio da Faculdade de Arquitetura, Engenharia e Tecnologia da Universidade Federal de Mato Grosso, UFMT. Na parte superior do prédio, o sensor foi fixado em uma plataforma de suporte onde já estava fixado o termo-higrômetro de referência para medição de temperatura e umidade relativa.

Para a realização da calibração dos sensores de temperatura e umidade relativa do termo-higrômetro necessitou realizar medidas comparativas com o termo-higrômetro de referência. $\mathrm{O}$ termo-higrômetro a ser calibrado e o de referência foram dispostos em mesmo ambiente sobre as mesmas condições. O período de avaliação foi de 48 horas, dias 4 e 5 de julho de 2016, com intervalo de tempo de 5 minutos.

Para desenvolvimento deste trabalho, o termo-higrômetro de referência é modelo $\mathrm{S}$ THB da marca OnsetComp com precisão de $0,2{ }^{\circ} \mathrm{C}$ para temperatura, resolução de 0,02 entre $0{ }^{\circ} \mathrm{C}$ a $50{ }^{\circ} \mathrm{C}$, e precisão de $2,5 \%$ para umidade relativa, resolução de $0,1 \%$ entre $10 \%$ a $90 \%$, com certificado de calibração.

O termo-higrômetro a ser calibrado é do modelo S-THB da marca OnsetComp com precisão de $0,2{ }^{\circ} \mathrm{C}$ para temperatura, resolução de 0,02 entre $0{ }^{\circ} \mathrm{C}$ a $50{ }^{\circ} \mathrm{C}$, e precisão de $2,5 \%$ para umidade relativa, resolução de $0,1 \%$ entre $10 \%$ a $90 \%$ (Figura 1 ).

Figura 1 - Termo-higrômetro calibrado

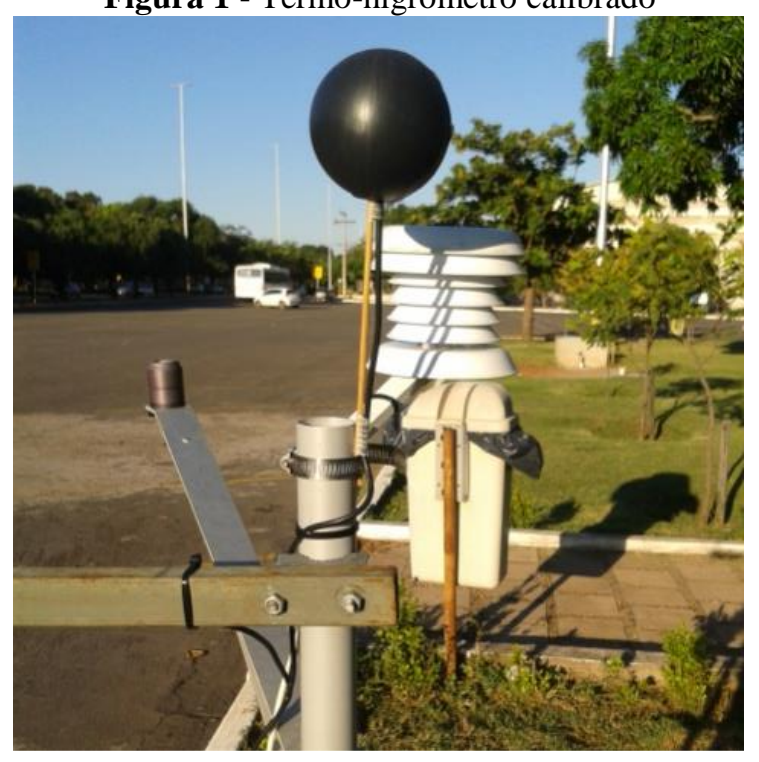

Os dados produzidos pelos sinais e/ou pulsos elétricos dos termo-higrômetros foram processados e armazenados por Datalloger, Marca OnsetComp, Modelo U30-NRC-VIA-10S100-000 (Figura 2). 
Guedes D. A. S.; Borges V. C. de A. L.; Calibração de Termohigrometro para Utilização em Pesquisas Microclimáticas. E\&S - Engineering and Science, (2017), 6:1.

Figura 2 - Estação meteorológica a ser instalada na parte superior do prédio

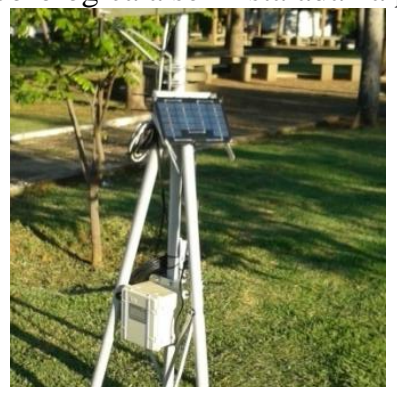

As medições geraram dados que foram armazenados no cartão de memória, em forma de arquivo texto (txt), tendo sido transferidos para um computador e convertidos para uma planilha eletrônica, para as análises estatísticas.

Após período de medidas comparativas, foi realizada a calibração dos sensores através de regressão linear simples (equação 1), obtendo-se os coeficientes angulares e lineares da reta e o coeficiente de determinação da reta. Foram realizadas 1443 medições simultâneas sendo os mesmos submetidos ao procedimento estatístico usando o programa de estatística do Excel

$$
\mathrm{Y}=\mathrm{a}+\mathrm{b} \cdot \mathrm{X}
$$

Em que Y é o valor obtido pelo termo-higrômetro calibrado e X é o valor obtido pelo termo-higrômetro de referência.

O nível de validação foi verificado pelos seguintes indicadores estatísticos: Erro em percentagem (\%), Erro Médio Absoluto (EMA), Raiz do Erro Quadrático Médio (REQM) e o Índice de Concordância (d).

O Erro em Percentagem (equação 2) é a representação percentual do erro relativo e permite a comparação de uma estimativa com um valor exato.

$$
\text { Erro }=\frac{P_{i}-O_{i}}{O_{i}} \times 100
$$

Em que $\mathrm{P}_{\mathrm{i}}$ é o valor obtido pelo termohigrômetro calibrado, $\mathrm{O}_{\mathrm{i}} \mathrm{o}$ valor obtido pelo termohigrômetro de referência.

O EMA (equação 3)indica o afastamento médio absoluto dos valores obtidos pelo termohigrômetro calibrado em relação aos valores obtidos pelo termohigrômetro de referência.

$$
\text { EMA }=\sum \frac{\left|P_{i}-O_{i}\right|}{N}
$$

Em que $\mathrm{N}$ indica o número de medições realizadas.

Já o REQM (equação 4) indica o quanto as medidas do termohigrômetro calibrado apresentam erros ao estimar a variabilidade das medidas em torno da média.

$$
\mathrm{REQM}=\sqrt{\frac{\sum\left(P_{i}-O_{i}\right)^{2}}{N}}
$$


Para determinar a exatidão que está relacionada ao afastamento dos valores obtidos pelo termo-higrômetro calibrado e o termo-higrômetro de referência, foi determinado o Índice de Concordância "d" (equação 5). Seus valores variam de 0 (zero), com nenhuma concordância, a 1 (um), com concordância perfeita.

$$
\mathrm{d}=1-\left[\sum\left(\mathrm{P}_{\mathrm{i}}-\mathrm{O}_{\mathrm{i}}\right)^{2 /} \sum\left(\left|\mathrm{P}_{\mathrm{i}}-\mathrm{O}\right|+\left|\mathrm{O}_{\mathrm{i}}-\mathrm{O}\right|\right)^{2}\right]
$$

Em que O é a média dos valores obtidos pelo termo-higrômetro de referência.

\section{RESULTADOS E DISCUSSÕES}

As medidas de temperatura e umidade relativa do termo-higrômetro calibrado foram bem próximas as medidas do termo-higrômetro de referência.

A Figura 3 representa os modelos ajustados por regressão linear. O coeficiente angular se aproxima de 1, o coeficiente de determinação $\left(\mathrm{R}^{2}\right)$ explica 99,9\% dos dados observados e o índice de concordância perfeito. Na Tabela 1, são apresentados os modelos ajustados por regressão linear.

Tabela 1 - Coeficiente linear (a) e angular (b) da regressão entre a temperatura (T) e umidade relativa do ar (UR) medidas pelo sensor de referência e pelo calibrado, coeficiente de correlação $(R)$, Índice de concordância $(d)$, erro médio absoluto $(E M A)$ e raiz do erro médio quadrático $(R E M Q)$.

\begin{tabular}{ccc}
\hline Variáveis & T & UR \\
\hline $\mathrm{a}$ & 0,161 & 1,538 \\
$\mathrm{~b}$ & 0,991 & 0,960 \\
$\mathrm{R}$ & 0,999 & 0,999 \\
$\mathrm{~d}$ & 1,000 & 1,000 \\
EMA & 0,103 & 0,718 \\
REQM & 0,128 & 0,949 \\
\hline
\end{tabular}

Figura 3 - Regressão linear entre o termo-higrômetro de referência e o termo-higrômetro calibrado.
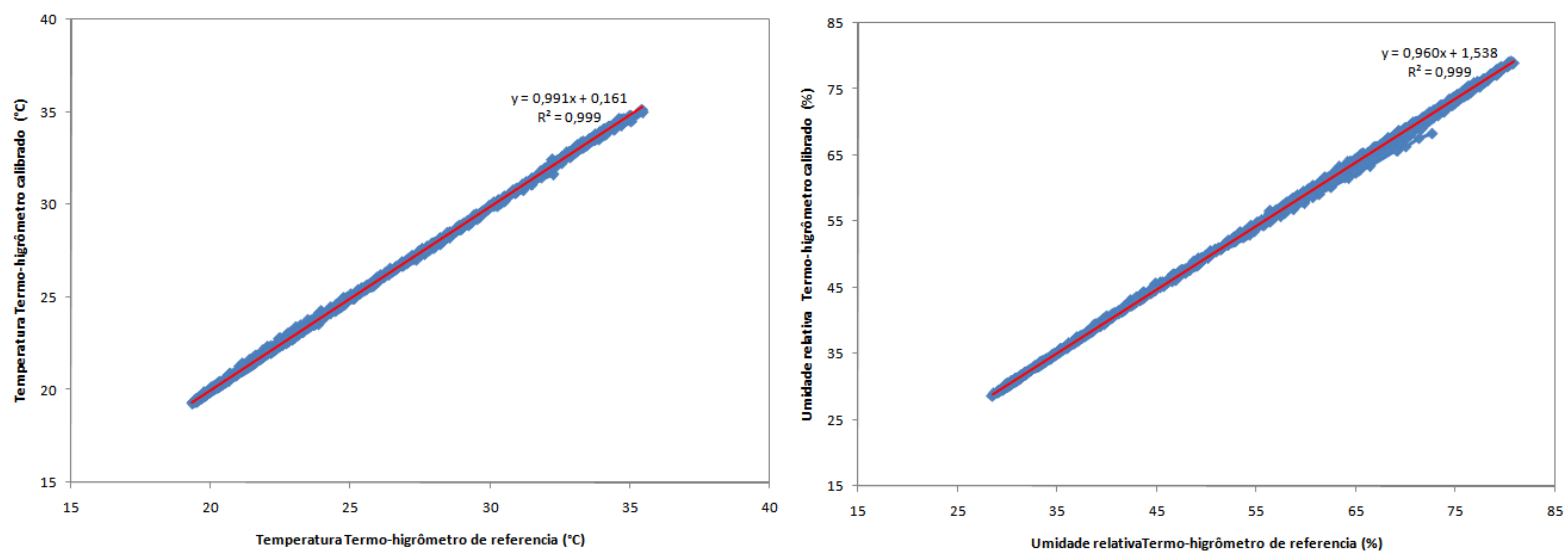

A correlação foi positiva indicando que o sensor foi calibrado corretamente.

Os resultados indicam uma ótima concordância entre os valores obtidos tanto para temperatura como para a UR do calibrado com o sensor de referência (Figura 2). Os valores máximos dos erros em percentagem obtidos foram de $1,56 \%$ e $2 \%$, para temperatura e umidade relativa respectivamente. Os valores mínimos foram de 1,90\% e 5,78\%.

A distribuição temporal dos dados obtidos pelo termo-higrômetro calibrado e o de referência para temperatura são observados na figura 4. Para o intervalo analisado nota-se que os picos de temperatura ocorriam entre $12: 00$ a 16 horas com valores entre 30 a $35,5{ }^{\circ} \mathrm{C}$. 
Graficamente os valores de temperatura medidos pelo sensor calibrado são poucos menores que os registrados pelo sensor de referência. As variações encontradas são mínimas, esse fato foi verificado estatisticamente pois, tanto os parâmetros da regressão como o coeficiente de determinação e a análise dos erros reforçam a validade da metodologia proposta nesse trabalho.

A distribuição temporal dos dados para UR são observados na figura 5. Observa-se que os valores de umidade relativa para o termo-higrômetro calibrado são ligeiramente inferiores ao termo-higrômetro de referência. Ambos sensores registraram valores próximos a 29\% para o dia 05 de julho de 2016 por volta das 15:00 horas. A máxima registrada para o termohigrômetro calibrado foi de 79,1\% e 80,5\% para o termo-higrômetro de referência no dia 05 de julho de 2016 por volta das 06:00 horas. Os valores mais elevados de UR, figura 5, foram registradas durante a madrugada, quando a temperatura do ar atinge seu valor mínimo, e mais baixo no início da tarde, correspondentemente ao máximo da temperatura de bulbo seco (Butera, 1995).

\section{CONSIDERAÇÕES FINAIS}

O termo-higrômetro calibrado apresentou medidas confiáveis de temperatura e umidade relativa pois estão próximos ao valor obtido pelo termo-higrômetro de referência.

O coeficiente de concordância foi igual a 1, obtendo uma concordância perfeita.

Foi obtido elevado coeficiente de determinação $\left(\mathrm{R}^{2}\right)$, que explica mais de $99 \%$ dos dados medidos como termo-higrômetro calibrado comparados com o termo-higrômetro de referência.

O termo-higrômetro calibrado mostrou-se confiável, podendo ser utilizado em pesquisas ambientais para análises de temperatura e umidade relativa em ambientes externos.

\section{REFERÊNCIAS}

Butera, F.M. Architettura e ambiente: manuale per ilcontrollo della qualitàtermica, luminosa e acústica de gliedifici. Milano: Etaslibri, 1995.

CAllejas, I. J. A. CONTRIBUiÇÃO AO processo de calibração de sensor de temperatura do tipo termistor com auxílio da plataforma arduíno. $2^{\circ}$ Encontro em Engenharia de Edificações e Ambiental, 2014

JENSEN, J.R. Sensoriamento remoto do ambiente: uma perspectiva em recursos terrestres. São José dos Campos: Parêntese, 2009. 604p.

NEVES, GAR et al. Desenvolvimento e Calibração de um Termohigrômetro para uso em Pesquisas de Micrometeorologia, Agrometeorologia e Climatológica. Revista Brasileira de Geografia Física V. 08 N. 01 (2015) p. 136-143.

SILVA, J.G. Monitoramento e Controle de Umidade e Temperatura em Secadores de Massa Alimentícias. 67f. Dissertação (Mestrado, Análise e Controle de Processos Químicos) Escola de Engenharia Mauá, Centro Universitário do Instituto Mauá de Tecnologia, São Caetano do Sul SP, 2009. 

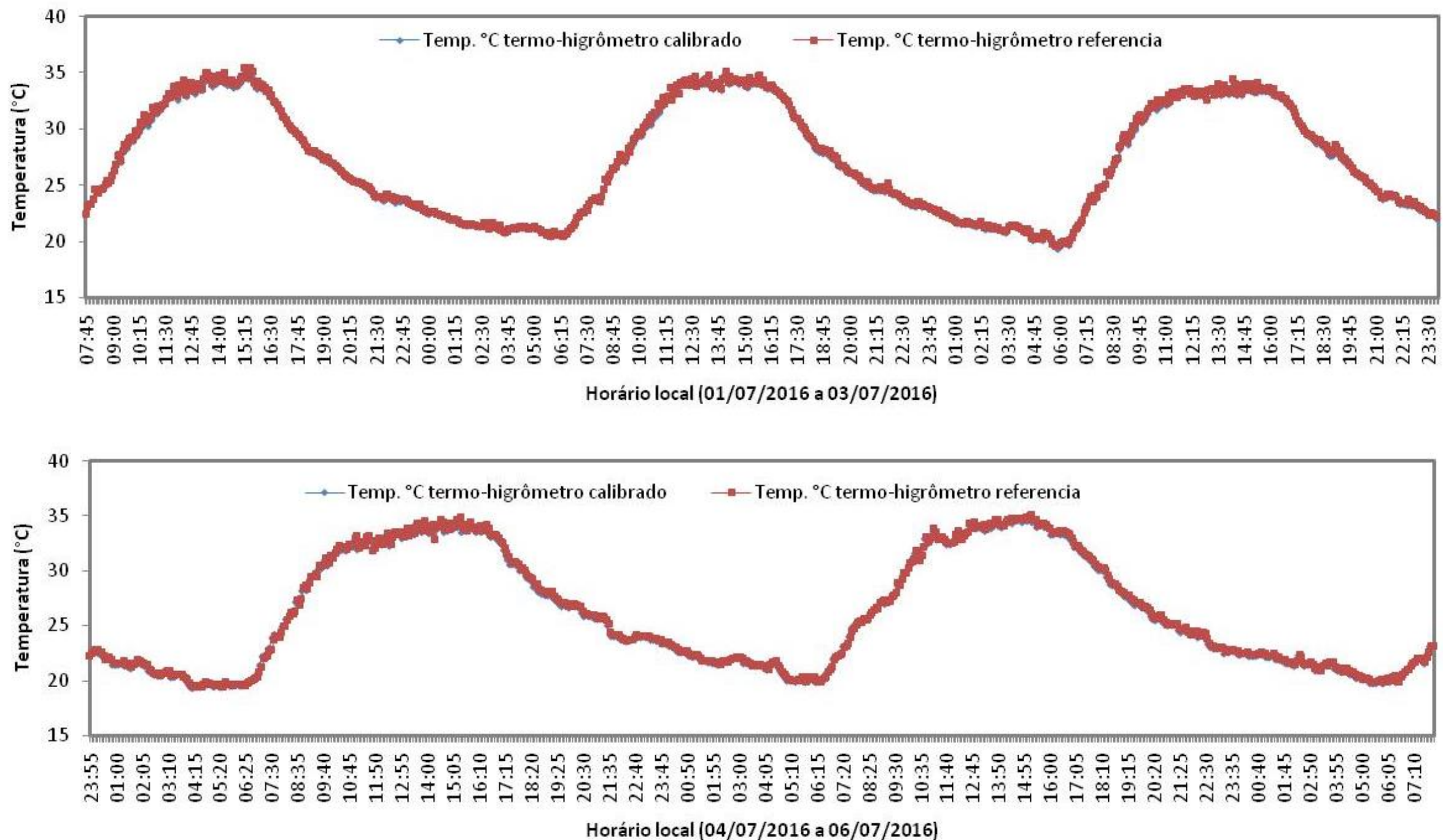

Figura 4 - Distribuição temporal da variável temperatura 

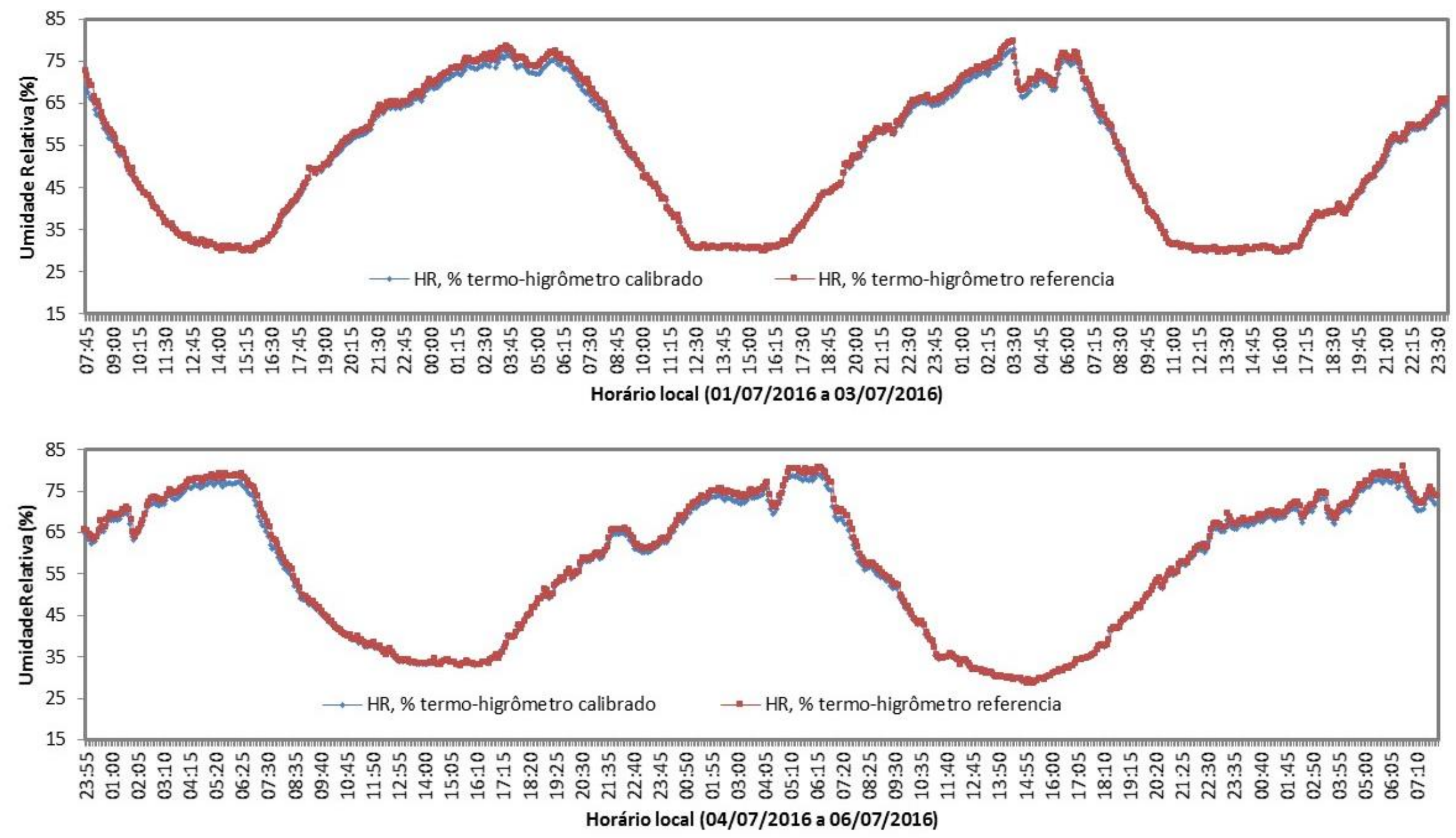

Figura 5 - Distribuição temporal da variável UR

E\&S - Engineering and Science ISSN: 2358-5390 DOI: 10.18607/ES20176071 Volume 1, Edição 6 